\title{
Heterotopic pregnancy: a case report
}

\author{
Tamer Ali ${ }^{1}$, Mohamed A. Tawab², Mona Abdel Ghaffar ElHariri ${ }^{*}$ and Alaa A. Ayad ${ }^{3}$
}

\begin{abstract}
Background: Heterotopic pregnancy describes the occurrence of two pregnancies in different implantation sites simultaneously, which is rare, yet it is a challenge to diagnose such a problem due to complex clinical and laboratory findings. In the current study, we present a case of first trimester heterotopic pregnancy diagnosed by ultrasound (US) and magnetic resonance imaging (MRI) and was managed successfully.

Case presentation: A 22-year-old primigravida lady with spontaneous pregnancy was presented by increasing lower abdominal pain for 5 days with brownish vaginal discharge, nausea, and vomiting episodes. Trans-abdominal and endovaginal ultrasound was performed and revealed a viable intrauterine pregnancy of 8 weeks and 1 day, associated with a heterogeneous complex right adnexal mass. MR imaging revealed a right adnexal mass intimately anterior to the normal right ovary. Laparascopy was done; it revealed a distended right fallopian tube with pregnancy while the right ovary was not seen (impeded in the pouch of Douglas), and right salpingectomy was done. The specimen was sent for histopathology. The patient tolerated the procedure well and was then taken to the recovery room in stable condition. The histopathological report confirmed the diagnosis of ectopic pregnancy.

Conclusion: However, heterotopic is a rare condition, any pregnant woman presenting with alarming abdominal pain and adnexal abnormality; heterotopic pregnancy should be among the differential diagnosis possibilities. The patient should be thoroughly investigated using ultrasound and MRI if needed, to exclude this rare diagnosis and allow on-time proper management.
\end{abstract}

Keywords: Heterotopic pregnancy, Gestational sac, Ultrasound

\section{Background}

Heterotopic pregnancy describes the occurrence of two pregnancies in different implantation sites simultaneously, mostly manifested as intrauterine and ectopic pregnancies (ampullary in 80\%). Heterotopic pregnancy is rare and estimated to occur in about 1 per 30,000 spontaneous pregnancies while a higher prevalence may occur in assisted reproduction techniques that may reach up to 1 case per 100 in some literatures. It is a challenge to diagnose such a problem due to complex clinical and laboratory findings [1-9].

In the current study, we present a case of first trimester heterotopic pregnancy diagnosed by ultrasound (US) and magnetic resonance imaging (MRI).

\footnotetext{
*Correspondence: Doctormona2000@yahoo.com

'Department of Radio-Diagnosis, Faculty of Medicine, Zagazig University, P.O. Box 184, Zagazig, Sharkia 44511, Egypt

Full list of author information is available at the end of the article
}

\section{Case presentation}

A 22-year-old primigravida lady with spontaneous pregnancy was presented by increasing lower abdominal pain for 5 days with brownish vaginal discharge, nausea, and vomiting episodes. Laboratory test showed a total $\beta$-HCG of $96,900 \mathrm{mIU} / \mathrm{mL}$.

Trans-abdominal and endovaginal ultrasound were performed using the Voluson E8 device (General Electric Healthcare, Austria) with a convex 2-5 and endocavitary $5-9 \mathrm{MHz}$ transducer, respectively. Grayscale ultrasound confirmed by color Doppler revealed a viable intrauterine pregnancy of 8 weeks and 1 day associated with a heterogeneous complex right adnexal mass (Fig. 1) suggestive of being a gestational sac. Pelvic fluid collection was also seen; otherwise, the ovaries displayed unremarkable ultrasound features. MRI imaging was done using Sonata Maestro Class 1.5 T device (Siemens, Erlangen, Germany). A phased-array surface coil is centered over the abdomen 

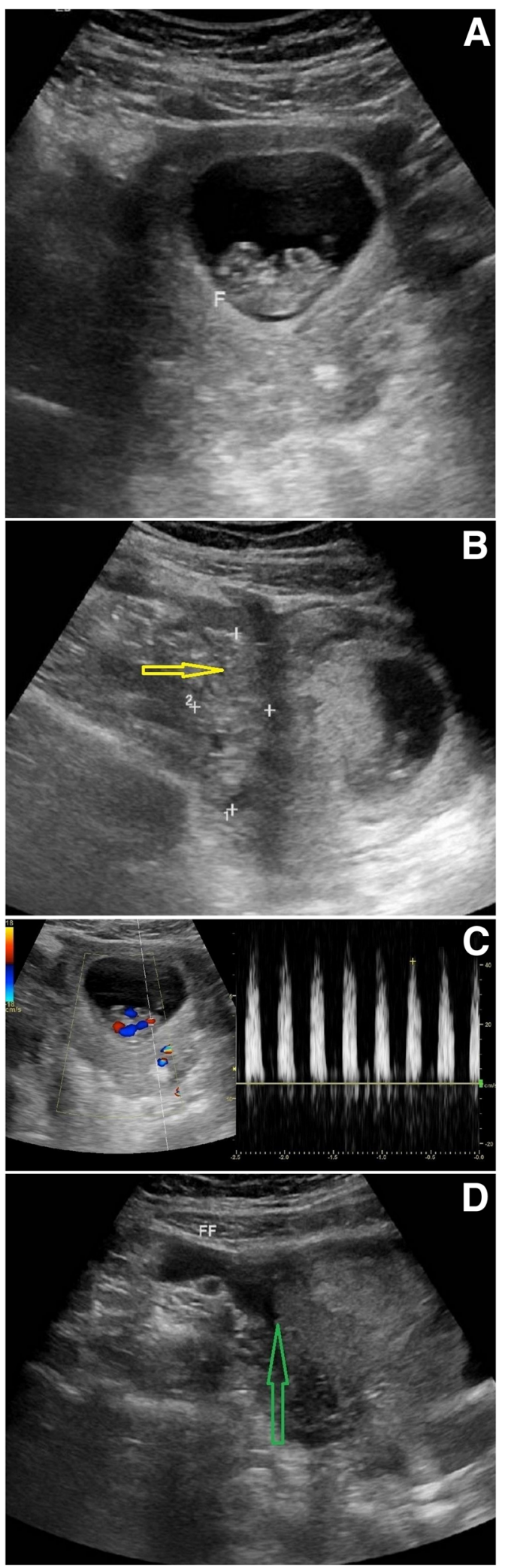

Fig. 1 a-d Ultrasound study shows the intrauterine fetus with cardiac pulsation and heterogenous right adnexal mass (yellow arrow), and pelvic fluid collection (green arrow) is noted

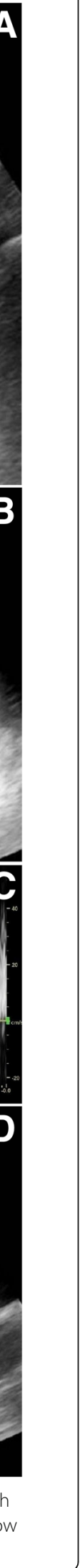

to cover till the symphysis pubis. Images are acquired from the level of the hepatic hilum to the symphysis pubis. The sequences included the turbo spin-echo (TSE) technique, gradient-echo (FLASH), and T1 and T2 weighting to obtain axial and coronal images. No contrast was given.

MR study revealed a right adnexal ovoid mass lesion $(29 \times 23 \times 17 \mathrm{~mm})$ intimately anterior to the normal right ovary, displaying a mixed hyper- and hypo-intense signal at T1 and T2 WI. It had a thick wall showing high T2 signal (Fig. 2).

After informed consent, the patient was transferred to the operating room where general anesthesia was applied. Laparoscopy was performed. It revealed distended right fallopian tube with pregnancy while the right ovary was not seen (impeded in the pouch of Douglas). Hemoperitoneum was noted while the left tube, left ovary, and the appendix were unremarkable. Right salpingectomy was done, and the specimen was sent to the histopathology. The patient had tolerated the procedure well.

Histopathological examination of the specimen showed a dilated congested segment of the right fallopian tube while microscopic examination revealed fragments of blood clot, decidual tissue, and chorionic villi with trophoblasts noted within the dilated fallopian tube consistent with tubal ectopic pregnancy.

\section{Discussion}

Ectopic pregnancy needs early diagnosis and management to avoid the high probability of tubal rupture. Heterotopic pregnancies can be asymptomatic in about half of the cases; otherwise, it can be presented by variable clinical presentations: mainly abdominal pain, adnexal swelling that may be associated with vaginal bleeding, or even shock due to hypovolemia. Unfortunately, the clinical findings are more frequently presented with tubal rupture [3-7].

While ectopic pregnancy can be early diagnosed during the screening of the serum b-HCG and endovaginal US in routine prenatal assessment, heterotopic pregnancy diagnosis can be a challenge as it may be delayed in the concurrence of intrauterine pregnancies. This can be due to the differential diagnosis between ectopic pregnancy and other situations that may be associated with normal pregnancy as hemorrhagic corpus luteum or adnexal torsion [2, 6-9].

In normal pregnancies, serum B-HCG above 1500$2000 \mathrm{mIU} / \mathrm{mL}$ should be associated with ultrasound visualized intrauterine (IU) pregnancy; however, this IU pregnancy will not necessarily exclude the chance of having a heterotopic pregnancy especially in cases receiving conception-assisted techniques.

Ultrasound picture of heterotopic pregnancy may be adnexal complex cysts or mass which can be explained by being hematosalpinx, tubal ring, or embryo. Free intra-peritoneal fluid can be seen also [4, 6-9]. 


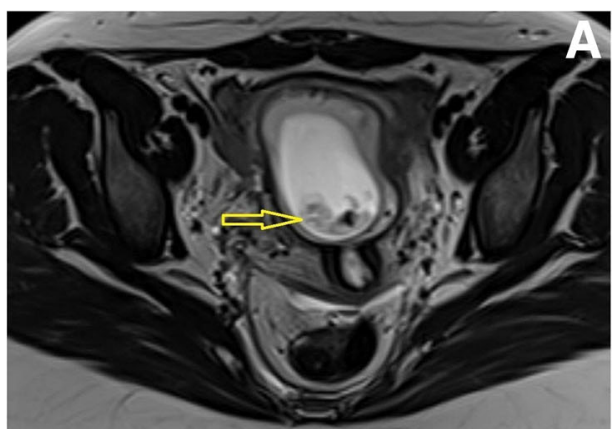

Fig. 2 MRI images (a, b: Ax T2; $\mathbf{c}$ d: T2 fat suppression) show the intrauterine fetus (yellow arrow) as well as a well-defined right adnexal lesion (blue arrow) with mixed signal with a high signal at its thick wall. Pelvic collection (green arrow). Note the normal right ovary (red arrow)
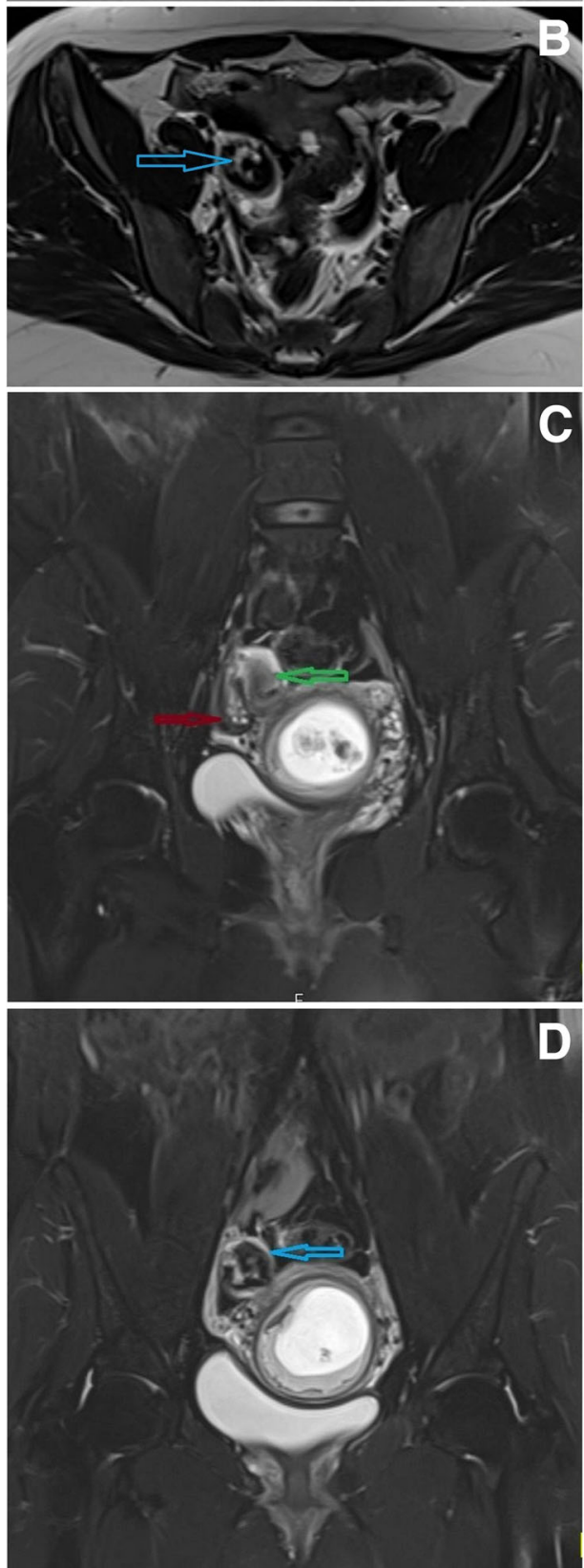

In equivocal cases, MRI can be helpful; it can show an adnexal lesion that may be cystic or looks like a gestational sac. Tubal cystic dilatation with a thickened wall can also be seen, while tubal rupture will cause hematoma [9].

Heterotopic pregnancy treatment needs laparoscopy and, most often, a salpingectomy or salpingostomy. However, in hemodynamically unstable cases, laparotomy may be needed. Systemic methotrexate has no role in the management of heterotopic pregnancy due to the presence of a viable intrauterine pregnancy. Some literature described the use of local injection of potassium chloride and methotrexate, but the success rate is controversial [10-14].

\section{Conclusion}

However, heterotopic is a rare condition, any pregnant woman presenting with alarming abdominal pain and adnexal abnormality; heterotopic pregnancy should be among the differential diagnosis possibilities. The patient should be thoroughly investigated using ultrasound and MRI if needed, to exclude this rare diagnosis and allow on-time proper management.

\section{Abbreviations}

US: Ultrasound; MRI: Magnetic resonance imaging; HCG: Human chorionic gonadotropin

\section{Acknowledgements}

Not applicable

\section{Authors' contributions}

AT conceived the study, participated in its design and coordination, drafted the manuscript, and carried out the radiological results. AM participated in the study design and helped in drafting the manuscript and radiological results. EM participated in the study design and helped in drafting the manuscript and radiological results. AA participated in the study design and helped in drafting the manuscript and radiological results. All authors read and approved the final manuscript.

\section{Funding}

Not applicable.

\section{Availability of data and materials}

The data that support the findings of this study are available on request from the corresponding author.

\section{Ethics approval and consent to participate}

This study was approved by the ethical committee of the institution as well as informed written consent from the patient included in this study was taken.

\section{Consent for publication}

All authors read and approved the final manuscript.

The patient included in this research gave written informed consent to publish the data contained within this study. 


\section{Author details}

'Department of Radio-Diagnosis, Faculty of Medicine, Zagazig University, P.O. Box 184, Zagazig, Sharkia 44511, Egypt. ${ }^{2}$ Department of Radio-Diagnosis, King Hamad University Hospital, Al Sayh, Bahrain. ${ }^{3}$ Department of Radiology, King Hamad University hospital, Al Sayh, Bahrain.

Received: 19 June 2020 Accepted: 22 September 2020

Published online: 23 October 2020

\section{References}

1. Fernandez H, Gervaise A (2004) Ectopic pregnancies after infertility treatment: modern diagnosis and therapeutic strategy. Hum Reprod Update 10(6):503-513

2. Callen PW Ultrasonography in obstetrics and gynecology. In: Levine D (ed) Ectopic Pregnancy, 5th edn. Saunders Elsevier, Philadelphia, pp 1020-1047

3. Xiao HM, Gong F, Mao ZH, Zhang H, Lu GX (2006) Analysis of 92 ectopic pregnancy patients after in vitro fertilization and embryo transfer. J Cent South Univ 31(4):584-587

4. Louis-Sylvestre C, Morice P, Chapron C, Dubuisson JB (1997) The role of laparoscopy in the diagnosis and management of heterotopic pregnancies. Hum Reprod 12(5):1100-1102

5. Jeon HS, Shin HJ, Kim IH, Chung DY (2012) A case of spontaneous heterotopic pregnancy presenting with heart activity of both embryos. Kor J Obstet Gynecol 55:339-342

6. Elito NAMD, Montenegro M, Costa Soares RDA, Camano L (2008) Unruptured ectopic pregnancy - diagnosis and treatment. State of art. Revista Brasileira de Ginecologia e Obstetricia 30(3):149-154

7. Dündar Ö, Tütüncü L, Müngen E, Muhcu M, Yergök YZ (2006) Heterotopic pregnancy: tubal ectopic pregnancy and monochorionic monoamniotic twin pregnancy: a case report. Perinatal J 14:96-100

8. Tamai K, Koyama T, Togashi K (2007) MR features of ectopic pregnancy. Eur Radiol 17(12):3236-3246

9. Li XH, Ouyang Y, Lu GX (2013) Value of transvaginal sonography in diagnosing heterotopic pregnancy after in-vitro fertilization with embryo transfer. Ultrasound Obstet Gynecol 41:563-569

10. Damario, Ectopic Pregnancy. In: Rock Jr, Jones III. Te Linde's Operative Gynecology. 10th. Philadelphia: Lippincott Williams and Wilkins; 2011. 794-795.

11. Li JB, Kong LZ, Yang JB, Niu G, Fan L, Huang JZ et al (2016) Management of heterotopic pregnancy: experience from 1 tertiary medical center. Medicine. 95:e2570

12. Baxi A, Kaushal M, Karmalkar H, Sahu P, Kadhi P, Daval B (2010) Successful expectant management of tubal heterotopic pregnancy. J Hum Reprod Sci 3:108-110

13. Deka D, Bahadur A, Singh A (2012) Neena Malhotra. Successful management of heterotopic pregnancy after fetal reduction using potassium chloride and methotrexate. J Hum Reprod Sci 5:57-60

14. Goldstein JS, Ratts VS, Philpott T, Dahan MH (2006) Risk of surgery after use of potassium chloride for treatment of tubal heterotopic pregnancy. Obstet Gynecol 107:506-508

\section{Publisher's Note}

Springer Nature remains neutral with regard to jurisdictional claims in published maps and institutional affiliations.

\section{Submit your manuscript to a SpringerOpen ${ }^{\circ}$ journal and benefit from:}

- Convenient online submission

- Rigorous peer review

- Open access: articles freely available online

- High visibility within the field

- Retaining the copyright to your article

Submit your next manuscript at $\boldsymbol{\nabla}$ springeropen.com 